\title{
Atomistic-scale simulations of the chemomechanical behavior of graphene under nanoprojectile impact
}

\author{
Kichul Yoon ${ }^{\mathrm{a}}$, Alireza Ostadhossein ${ }^{\mathrm{b}}$, Adri C.T. van Duin ${ }^{\mathrm{a},{ }^{*}}$ \\ ${ }^{a}$ Department of Mechanical and Nuclear Engineering, The Pennsylvania State University, \\ University Park, PA 16802, USA \\ ${ }^{\mathrm{b}}$ Department of Engineering Science and Mechanics, The Pennsylvania State University, \\ University Park, PA 16802, USA
}

${ }^{*}$ Corresponding author.

Tel: +1 814863 6277. E-mail address: acv13@psu.edu (Adri C.T. van Duin) 


\section{ABSTRACT}

Despite its importance, the mechanical behavior of graphene under the impact of projectiles has rarely been studied due to experimental and computational difficulties. Here, we simulated the impact of silica and nickel projectiles with a supersonic initial velocity on graphene. Then we analyzed the impact by using ReaxFF reactive force field method, which is capable of describing the entire system. During the process of projectile penetration, we identified various atomistic features, such as the formation of pentagon/heptagon pairs at the edges of the cracks, and the preferential crack edges that are affected by the deformability of graphene before crack initiation. Effects of defects in graphene and the material type of the projectile on specific penetration energy $\left(E_{p}{ }^{*}\right)$ also were addressed. The values of $E_{p}{ }^{*}$ obtained in our simulations were in general agreement with the recent experimental values reported by Lee

et al. [Science 2014, 346, (6213), 1092-1096]. Our simulation results showed that $E_{p}{ }^{*}$ was correlated with the diameter of maximum deformation of graphene before crack initiation, demonstrating the superior $E_{p}{ }^{*}$ of graphene as a result of its high ultimate stress and strain.

\section{Introduction}

Since the discovery of graphene [1], which has an ideal, two dimensional, hexagonal network of covalently-bonded carbon atoms, significant attention has been focused on graphene's remarkable mechanical, electronic, thermal, and chemical properties [2-12]. Specifically, graphene's superior intrinsic strength and Young's modulus, exceeding $100 \mathrm{GPa}$ and $1 \mathrm{TPa}$ [5], respectively, have made graphene and graphene-based nano-composites attractive in applications that require superstrong structural materials [13]. However, most studies of the mechanical behavior of graphene have been limited to the tensile properties, and only a few studies exist that 
address the mechanical behavior of graphene under the impact of projectiles [14-17]. The scarcity of such studies is due mostly to the difficulties and limitations encountered in conducting the experiments and doing the computations. From an experimental perspective, it is extremely challenging to prepare a large scale, free-standing graphene with uniform thickness, to control the speed of particles, and to characterize the dynamic response of the impact. From a computational perspective, ab initio as well as tight binding methods for the dynamic modeling of graphene under the impact of particles are very expensive, although they have been successful in the prediction of the mechanical properties of graphene [18-21]. Reactive force field methods, which also have been successful in the study of the mechanical behavior of graphene [20, 22-28], are less expensive and thus affordable candidates for studying the dynamic mechanical behavior of graphene in extreme environments. Especially, ReaxFF reactive force field methods, which are transferable to a wide range of materials, are a relevant tool for this research. Successful applications of ReaxFF reactive force field in the study of the mechanical behavior of graphene can be found in several previous studies [16, 29-35]. It should be noted, however, that, in the recent work by Jensen et al. [35], the carbon parameters (ReaxFF C-2013) predicted nonlinearity at moderate strain in the tensile curves of graphene, which led to inaccurate elastic constants. In this regard, the tensile properties of graphene seem to be better predicted by the potentials of REBO family, which are well-known reactive potentials for purely carbonaceous system [36, 37]. Still, the ultimate stress and strain predicted by the ReaxFF C-2013 were in good agreement with experimental and DFT data. Therefore, we used the ReaxFF reactive force field here, since it can also describe the interaction between graphene and nanoparticles, which is currently not supported in the REBO family. Furthermore, prediction of vacancy energies by the ReaxFF reactive force field showed good agreement with DFT data $[38,39]$. We believe that the 
non-linearity in the tensile curves does not significantly affect our impact simulations because the crack initiation and extension are mostly related to the finite strain region (large deformation region).

In 2014, Lee et al. [14] conducted an interesting set of experiments in which they discharged a micrometer-sized projectile with a supersonic initial velocity towards multi-layered graphene, approximately 30-300 layers, to examine the dynamic mechanical behavior of graphene involving high-strain-rate deformation. They calculated the loss of kinetic energy of the projectile by measuring its velocity before and after the penetration. Specific penetration energy $\left(E_{p}{ }^{*}\right)$, which is defined as the mass-specific energy that graphene absorbs from the projectile's loss of kinetic energy, was reported as more than ten times greater than that of macroscopic steel sheets. However, they did not capture the atomistic features on the fractures or the effects of defects and the material used to make the projectile.

Here, we simulated the penetration of graphene by nanometer-sized silica and nickel projectiles with a supersonic velocity, using the ReaxFF reactive potential. We investigated the evolution of the system around the cracks and identified preferential crack edges by counting the armchair and zigzag edges. In addition to the structural analysis, we investigated the effect of defects on $E_{p}{ }^{*}$ by introducing defects, such as mono-vacancies and grain boundaries in the graphene. We also explored how the type of material used to make the projectile affected the cracks' features and $E_{p}{ }^{*}$ by comparing the penetration results obtained with different projectile materials. We finally elucidated the important factors that give rise to the superior $E_{p}{ }^{*}$ of graphene.

\section{Computational methods and details}


ReaxFF [40] is a general bond-order-based potential that can describe connectivity changes smoothly. Bond orders are calculated based on inter-atomic distances at every iteration step to determine the changes in the connectivity of the particles. Accordingly, valence and torsion angles as well as chemical bonds are treated properly. This allows the accurate description of the chemical reactions that consist of bond formation and dissociation. In addition, ReaxFF considers non-bonded interactions such as van der Waals and Coulomb interactions with excessive short-range interactions avoided by using a shielding term in both potential terms, ensuring more realistic simulation. A detailed description of ReaxFF can be found elsewhere [ 40 , $41]$.

Molecular dynamic (MD) simulations in microcanonical ensemble (NVE) were performed using ReaxFF, which was implemented in the Large-scale Atomic/Molecular Massively Parallel Simulator (LAMMPS) package [42]. For penetration simulations by the silica and nickel projectiles, we integrated the ReaxFF C-2013 carbon parameters [38], which were trained to reasonably reproduce the mechanical properties of carbon materials, with previously published $\mathrm{Si} / \mathrm{O} / \mathrm{C} / \mathrm{H}$ [43] and Ni/O/C/H ReaxFF parameter sets [44]. A further description of these force fields used in this research is described as follows.

The ReaxFF $\mathrm{Si} / \mathrm{O} / \mathrm{C} / \mathrm{H}$ description used here was obtained based on the previous force fields developed for the carbon [38] and silica [45] descriptions. Additionally, the $\mathrm{Si} / \mathrm{C}$ interactions were incorporated in the previous $\mathrm{ReaxFF} \mathrm{Si} / \mathrm{O} / \mathrm{C} / \mathrm{H}$ description by fitting it against binding energy, heat of formation, and $\mathrm{EOS}$ for $\mathrm{SiC}$ crystals in addition to the $\mathrm{Si}-\mathrm{C}$ bond dissociation energy profile. The carbon parameters [38] were developed by Srinivasan et al. based on a new set of quantum calculations consisting of equation of state (EOS) for graphite and diamond and the formation energies of various defects in graphene. The carbon parameters 
successfully predicted the energy barrier for Stone-Wales transformation in a $\mathrm{C}_{60}\left(I_{h}\right)$ fullerene. The silica parameters [45], developed by Fogarty et al., were fit against the quantum mechanical data such as EOS for bulk $\mathrm{Si}$ and $\mathrm{SiO}_{2}$ as well as bond dissociation curves and angle distortion energies for $\mathrm{Si} / \mathrm{O} / \mathrm{H}$ combinations.

The ReaxF Ni/O/C/H description used in this work was acquired by incorporating the Srinivasan et al. carbon [38] and the Zou et al. Ni/O [46] descriptions into the Mueller et al. $\mathrm{Ni} / \mathrm{O} / \mathrm{C} / \mathrm{H}$ force field [44]. The Mueller et al. $\mathrm{Ni} / \mathrm{C} / \mathrm{H} / \mathrm{O}$ force field was parameterized previously against the quantum dataset comprising EOS, the heat of formation for $\mathrm{Ni}$ and $\mathrm{NiC}$, the binding energies of hydrocarbons on various $\mathrm{Ni}$ crystal surfaces, and the binding characteristics of $\mathrm{Ni}$ to hydrocarbon fragments. The $\mathrm{Ni} / \mathrm{O}$ parameters were derived from EOS for $\mathrm{Ni}$ and $\mathrm{NiO}$ and vacancy formation energy in $\mathrm{Ni}$ crystals. The parameter sets for $\mathrm{Si} / \mathrm{O} / \mathrm{C} / \mathrm{H}$ and $\mathrm{Ni} / \mathrm{O} / \mathrm{C} / \mathrm{H}$ descriptions can be found in the supporting information.

The graphene sheets were constructed with approximately $380 \AA \times 360 \AA$, and they were hydrogenated to passivate the edges. Mono-vacancies were introduced into the pristine graphene sheets by removing carbon atoms from their original sites, followed by $0.1 \mathrm{~K} \mathrm{MD}$ minimization and subsequent $300 \mathrm{~K}$ equilibration steps. It should be noted that mono-vacancy is stable when it is isolated, but it is known to migrate and coalesce into a larger defect at slightly higher than room temperature $[47,48]$. However, in the time-scale (ps) of this study, coalescence of monovacancies was not observed during the equilibration. We used the model of graphene with monovacancies to address the effect of the defect density on the mechanical behavior of graphene under the impact simulations. An amorphous silica structure was prepared by annealing crystalline $\alpha$-quartz at around $3500 \mathrm{~K}$, with a significantly low cooling rate to ensure less defects in the final structure. A spherically-shaped silica projectile was excavated from the amorphous 
silica structure. The surface of the silica projectile was passivated by hydroxyl groups through separate hydroxylation modeling in water at $500 \mathrm{~K}$, such that the dangling bonds at the surface were saturated. The diameter of the final silica projectile after hydroxylation was determined to be $48 \AA$. The nickel projectile was constructed from fcc nickel crystal. The spherically-cut fcc nickel crystal was annealed at around its melting point, i.e. $1728 \mathrm{~K}$, by slowly ramping up from room temperature and down to room temperature. In order to make an inert surface of nickel, the annealed nickel sphere was oxidized at room temperature until the dangling bonds were saturated. For accurate comparison of $E_{p}{ }^{*}$ values, we matched the masses of the projectiles after the passivation processes to keep the kinetic energy the same at the same initial impact velocity. The diameter of the final oxidized nickel projectile was $38 \AA$.

To set up our simulations, the entire system was equilibrated at $300 \mathrm{~K}$ prior to the MD simulations. An initial velocity was set for the projectile, while the edges of the hydrogenated graphene sheet were fixed to imitate the experimental settings. We set the initial velocity as 5 $\mathrm{km} / \mathrm{s}$, which was within the realistic range of projectile speeds, and the time step as 0.05 fs to ensure the conservation of the total energy in the extremely dynamic conditions. In this study, the size of the projectile could not be matched to the size in the experiments due to the computational limit. Therefore, in order to impose high enough energy on the projectile for the penetration of graphene, we chose $5 \mathrm{~km} / \mathrm{s}$ as the initial velocity, which is larger than the velocities, $\sim 1 \mathrm{~km} / \mathrm{s}$, used in the experiments. The results were compared with the expected experimental results for multilayer graphene, provided by Lee et al.

\section{Results and discussion}


During the penetration process, the kinetic energy of the projectile $\left(E_{k i n, \text { projectile }}\right)$ decreases while the potential energy $\left(E_{\text {pot, projectile }}\right)$ increases due to the deformation, leading to a decrease in the total energy of the projectile $\left(\Delta E_{\text {projectile }}<0\right)$. This decrease is equal to the total energy increase of the graphene sheet $\left(\Delta E_{\text {graphene }}>0\right)$. The total energy change of the graphene sheet before and after penetration, which is called the penetration energy $\left(E_{p}\right)$, was calculated to evaluate the specific penetration energy $\left(E_{p}{ }^{*}\right)$.

$$
E_{p}=\Delta E_{\text {graphene }}=-\Delta E_{\text {projectile }}=-\left(\Delta E_{\text {kin, projectile }}+\Delta E_{\text {pot, projectile }}\right)
$$

Specific penetration energy is expressed by $E_{p}{ }^{*}=E_{p} / \rho A_{s}$, where $\rho$ is the surface density of graphene, and $A_{s}$ is the impact area, $\pi D_{\text {deformed }} / 4$, where $D_{\text {deformed }}$ is the deformed diameter of the projectile. In the simulations with nanometer-sized projectiles, unlike in the experiments by Lee et al. [14], we observed a significant deformation of the projectile, and thus we use $D_{\text {deformed }}$ in the calculation of the impact area, $A_{s}$.

The atomistic structures in Figs. 1(a)-(d), taken at the specific times indicated in Fig. 1(e), show the structural evolution during the penetration of the pristine graphene by the silica projectile. An important feature in the penetration process is that the energy required to extend the cracks comes from the kinetic energy of graphene as well as the energy released by relaxation of graphene, as explained in the following. From time $b$ to time $c$ in Fig. 1(e), when most of cracks have been extended, the potential energy of the graphene keeps increasing even during relaxation of graphene outside the fracture, while the total energy of the graphene remains constant, which indicates that the energy for the increase of potential energy originates from the kinetic energy of graphene. We observed two other structural relaxation periods between time $a$ and time $b$ and between time $c$ and time $d$. The first structural relaxation occurred when the graphene yielded an opening for the projectile to pass through. As the projectile started to 
penetrate the graphene, the significantly as-deformed graphene was relaxed and the potential energy decreased. The second structural relaxation occurred after time $c$. Comparison of Fig. 1 (d) with Fig. 1(c) clearly indicates that the opening became smaller as time progressed. In order to show the interaction between graphene and projectile at the initial stage of the penetration process, side-view snapshots at times $a$ and $b$ were provided in Fig. S5. The structural evolution of the silica projectile also was analyzed in Fig. 1(f) by using the eccentricity parameter, expressed as $e=\left(1-r_{b}^{2} / r_{a}^{2}\right)^{1 / 2}$, where $r_{a}$ and $r_{b}$ are the lengths of major and minor axes of the ellipse, respectively. $r_{a}$ and $r_{b}$ were determined based on the position of atoms, located at the edges of projectile. The spherical shape of the projectile had an eccentricity of zero. The maximum $e$, which was determined to be 0.78 , implied that the deformation of projectile was so significant that it cannot be ignored in the calculation of $E_{p}{ }^{*}$. The deformation of the silica projectile was not completely elastic, as indicated in Fig. 1(f), in which $e$ of the silica projectile converged into 0.6. The graphene sheets used in the impact simulation seem to be large enough and do not seem to produce adverse effects on the results, considering the facts described in the following: first, the cracks were initiated before the projectile-induced deformation reached the edges; second, until time $a$ when the interaction between graphene and projectile was almost over, the deformation did not reach the edges yet, indicating that no major edge effect was imposed to the calculation of energies in Fig. 1(e). 

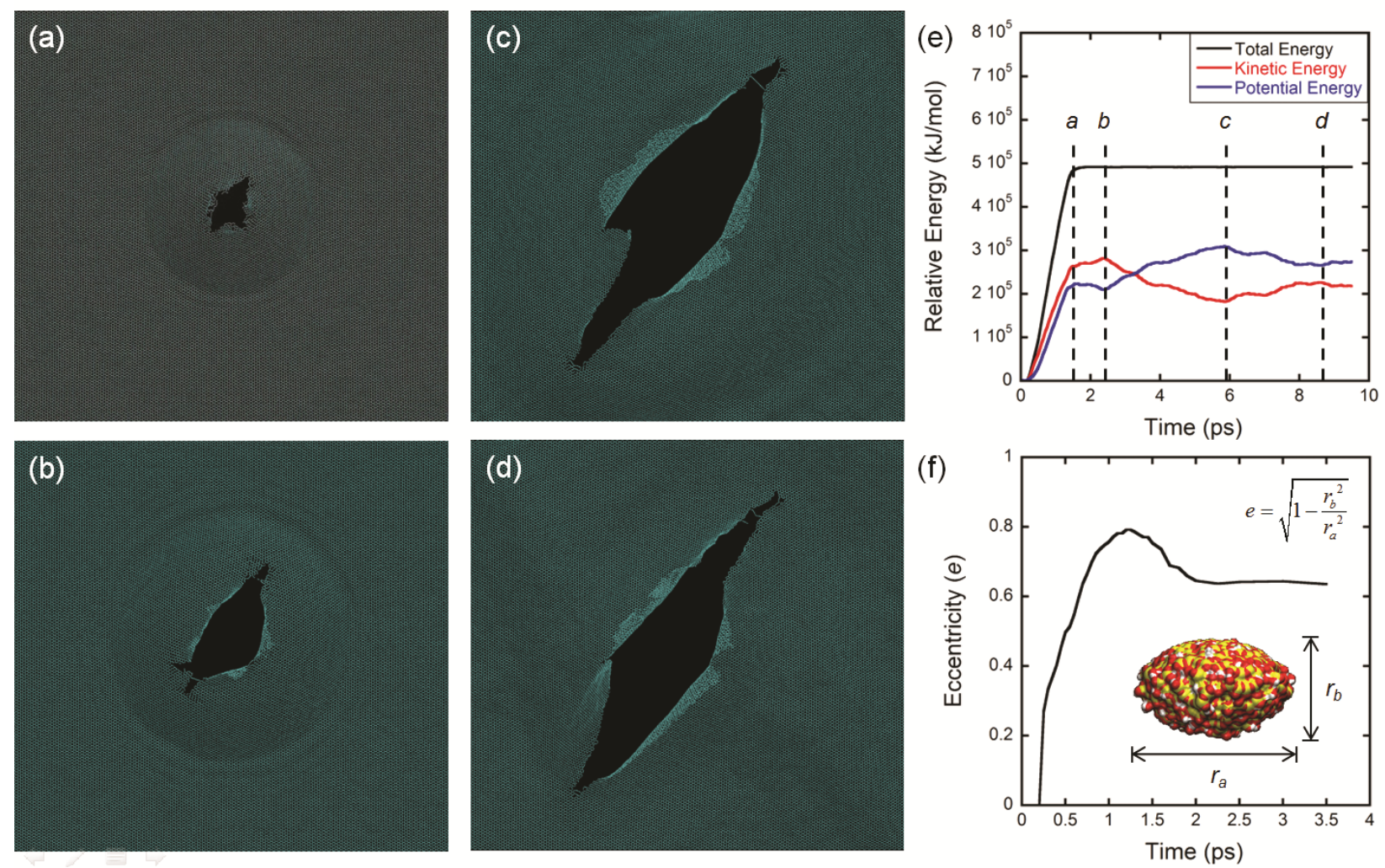

Figure 1. Evolution of the atomistic structures and energy during the penetration of pristine graphene by the silica projectile: (a)-(d) Atomistic structures of graphene taken at times $a-d$, indicated in (e); (e) Energy evolution of graphene during the penetration; (f) Structure evolution of the silica projectile; deformation of the projectile was expressed by using the concept of eccentricity, defined as $e=\left(1-r_{b}^{2} / r_{a}^{2}\right)^{1 / 2}$. The inset shows the silica projectile at maximum deformation. (yellow atoms: silicon; red atoms: oxygen; white atoms: hydrogen)

Penetration of pristine graphene by the nickel projectile exhibited different characteristics in the structural evolution but similar characteristics in the energy change upon penetration. Figs. 2(a)-(c) show that some constituents of the projectile remained at the surface of the crack, which was contrary to the case of the silica projectile, indicating that the reaction occurred between the projectile and surface of the graphene. The most frequently observed bonds between the constituents and the graphene are shown in Fig. 2(d), in which nickel atoms are bonded to one or 
two unsaturated carbon atoms at the edges of the cracks. The gentle slope of the potential energy of graphene at around time $a$ is an indication of the first structural relaxation of graphene. Most of the cracks were propagated between time $a$ and time $b$, and we observed the increase in the potential energy of graphene during that period. After time $c$, the decrease in the potential energy of graphene was related to the structural relaxation and the healing of defects, as evidenced by comparing Fig. 2(c) and Fig. 2(b). In the latter figure, the opening caused by the penetration of the projectile indeed became smaller than it was at time $b$. The maximum deformation of the nickel projectile $(e=0.48)$ was much less than that of the silica projectile $(e=0.78)$. Due to the loss of nickel and oxygen atoms from the projectile, the eccentricity of the nickel projectile after the maximum deformation was not observed. The smaller deformation of the nickel projectile can be explained by the fact that the cracks were initiated earlier, and they seemed to originate from the weakened mechanical properties of the graphene caused by the interaction between nickel and graphene. Figs. S1 and S2 show that the maximum deformation diameters in the penetrations by the nickel projectile always were much smaller than those caused by the silica projectile. 

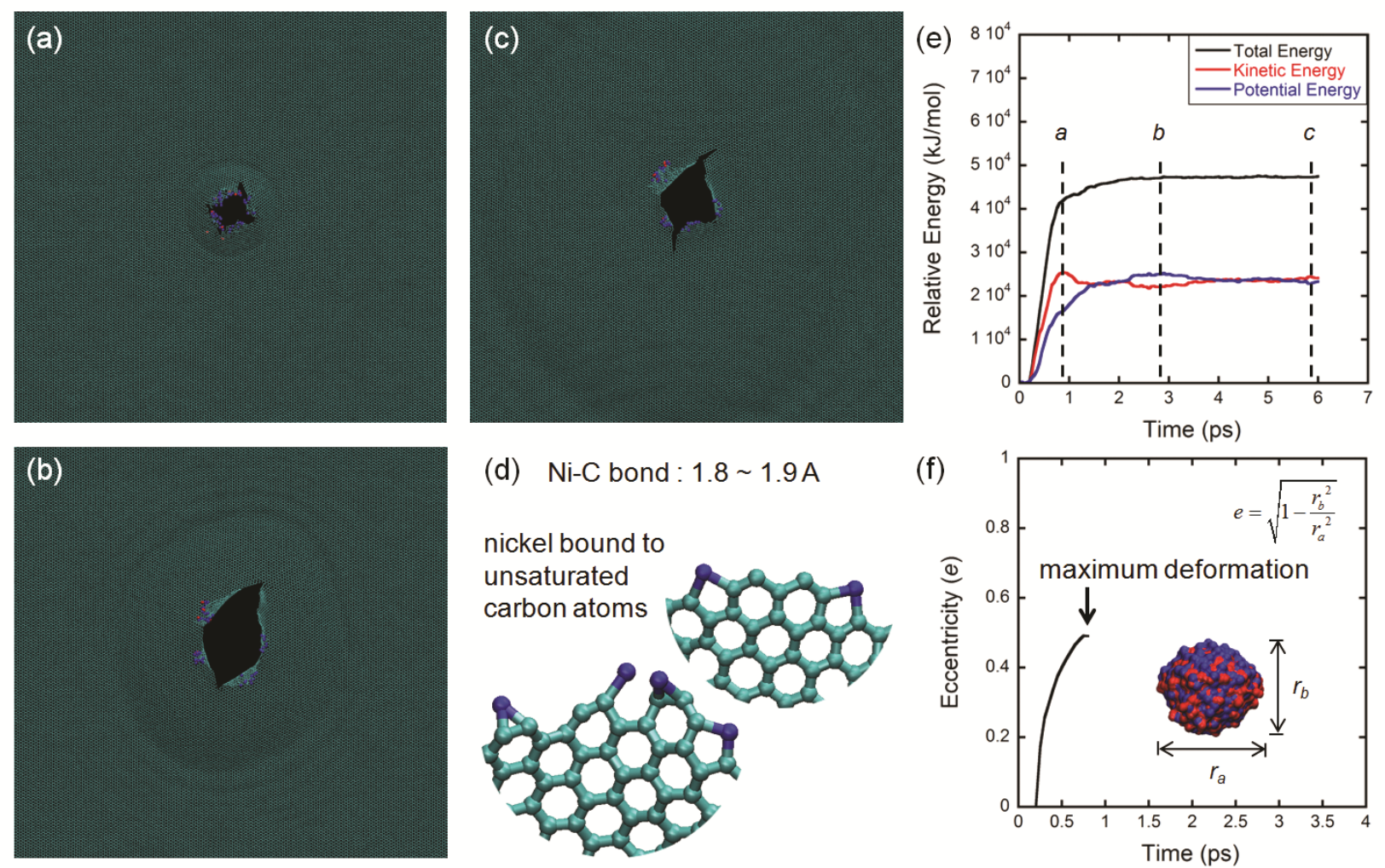

(d) Ni-C bond: $1.8 \sim 1.9 \mathrm{~A}$
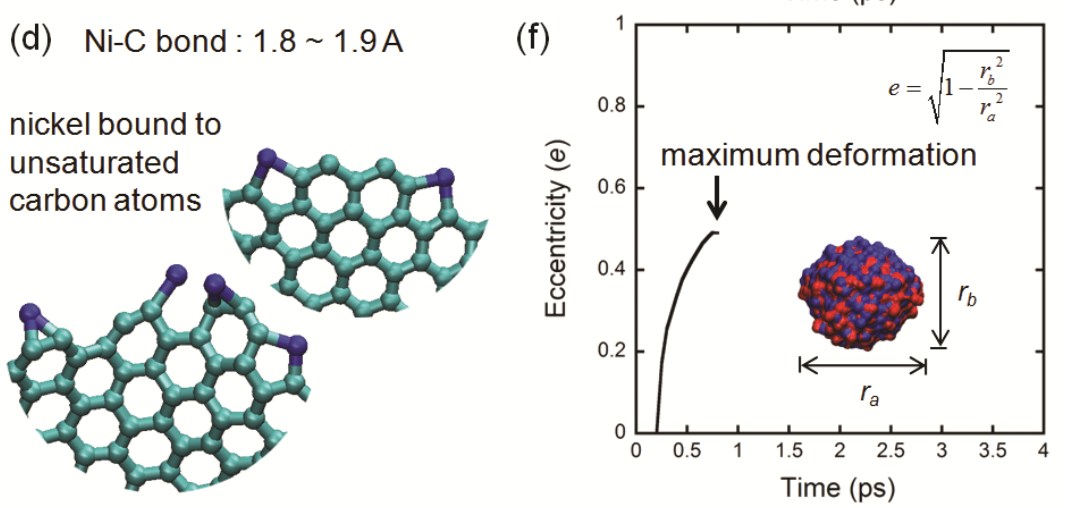

Figure 2. Evolution of the atomistic structures and energy during the penetration of pristine graphene by the nickel projectile: (a)-(c) Atomistic structures of graphene taken at times $a-c$, indicated in (e); (d) Most frequently found bonds between nickel and carbon atoms; (e) Energy evolution of graphene during the penetration; (f) Structure evolution of the nickel projectile: deformation of the projectile is expressed by using the concept of eccentricity, defined as $e=(1$ $\left.r_{b}^{2} / r_{a}^{2}\right)^{1 / 2}$. The inset shows the nickel projectile at maximum deformation. Eccentricity was obtained until the maximum deformation of the projectile, after which it became unclear how to define $r_{a}$ and $r_{b}$ due to loss of nickel and oxygen atoms from the projectile. (Blue atoms: nickel; red atoms: oxygen)

Looking at the crack atomistic-scale features in more detail, we identified several similarities and several differences between the penetrations by the silica and nickel projectiles. In both cases, pairs of pentagon/heptagon (5/7) defects were observed at the edges of the cracks, 
as the result of edge reconstruction, and seven-member rings almost always were found to be located closer to the tips of the cracks, as indicated in Fig. 3. These edge characteristics were consistent in the penetration of graphene with different levels of defects. These findings agree with the results reported by Terdalkar et al. [49], and they can be explained by the formation mechanism of 5/7 defects (consisting of bond breaking of the tips of the cracks, followed by the bond rotation) and by their favorability in facilitating structural relaxation and the release of energy. The stability of 5/7 defects in graphene edges was also reported by Koskinen et al., and it was the result of their structures which make the hybridization energy cost lower [50]. Significant differences were observed along the preferential edges along the cracks. The penetration of graphene by the silica projectile, shown in Fig. 3(a), led to the formation of more armchair edges (54\%) than zigzag edges (34\%). This observation was in good agreement with the previously reported data about the thermodynamic stability of armchair edges in the tearing of graphene $[29,30]$. However, in the case of penetration by the nickel projectile, the zigzag edges $(40 \%)$ were the most frequently observed kind of edges. We hypothesized that the reason for the difference was the earlier initiation of cracks when the graphene was penetrated by the nickel projectile, due to the nickel-graphene interactions that weakened the mechanical properties of graphene. Therefore, the cracks did not seem to be propagated along the thermodynamically more stable path which is the armchair direction. The ratios of armchair edges to zigzag edges, indicated in Fig. S3 and S4, support our hypothesis. Indeed, in all the penetrations of graphene by the nickel projectile, more zigzag edges, compared to the armchair edges, were observed. Furthermore, in the penetration by the silica projectile, the graphene with the mono-vacancy density of 0.036 and $0.04 \%$ yielded more zigzag edges, and the difference 
seems to be quite significant. All of the atomistic configurations of graphene after the penetrations are presented in Fig. S3 and S4 in the Supporting Information.
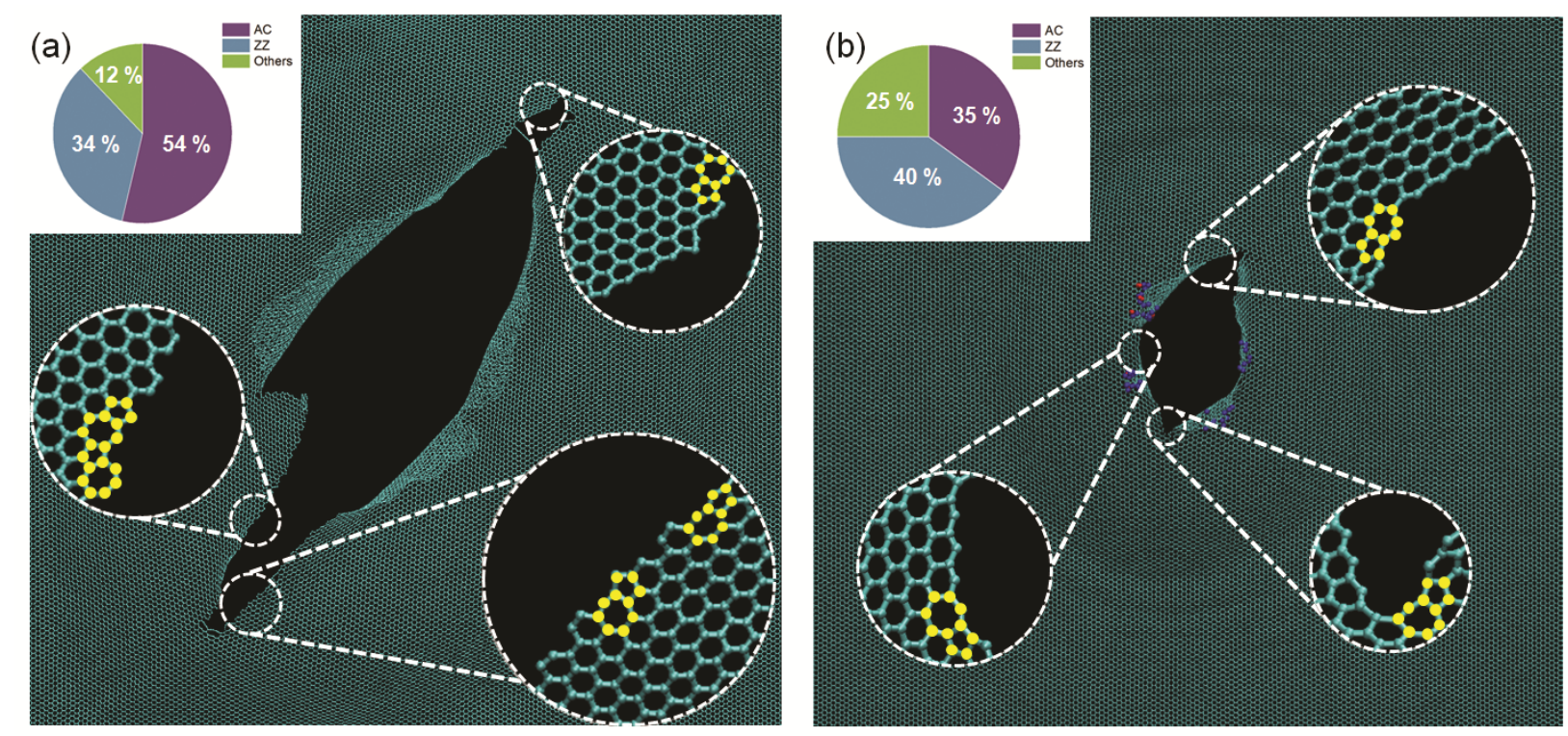

Figure 3. Atomistic features of penetration-induced cracks: The insets in (a) and (b) show percentage of crack edge orientations and magnified atomistic structures that indicate crack edge directions as well as formation of 5/7 Stone-Wales defects (indicated as yellow circles). (a) An atomistic structure obtained after the penetration by the silica projectile; (b) An atomistic structure obtained after the penetration by the nickel projectile

Figure 4 (a) shows the effects of defects and the type of material the projectile was made of on $E_{p}{ }^{*}$. Clearly, the trend of $E_{p}{ }^{*}$ with respect to the number of mono-vacancies in the penetration by the silica projectile was different from that in the penetration by the nickel projectile. For the penetration by the silica projectile, $E_{p}{ }^{*}$ decreased slightly as more monovacancies were introduced outside the impact region. However, when an additional monovacancy was introduced inside the impact region, $E_{p}{ }^{*}$ decreased by about $35 \%$. A larger number of mono-vacancies inside the impact region do not seem to significantly change $E_{p}{ }^{*}$. However, 
the penetration by the nickel projectile showed a monotonous decrease in $E_{p}{ }^{*}$ as more vacancies were introduced, irrespective of the location of the vacancies.

The decrease in $E_{p}{ }^{*}$ due to the introduction of defects appears to originate from the less allowable deformation of graphene before the initiation of the cracks, as shown in Fig. 4(b). In the penetrations by both the silica and the nickel projectiles, the maximum deformation diameter $\left(D_{\max }\right)$ decreased when the concentration of vacancies increased, and $E_{p}{ }^{*}$ had a linear dependence on $D_{\max }$ normalized by the diameter of the projectile $(D)$, indicating that $E_{p}{ }^{*}$ was affected by the deformability of graphene before the initiation of the cracks. Figs. S1 and S2 show the atomistic structures of graphene before the cracks were initiated and their $D_{\max }$ values. We conclude that a large allowable deformation and a large deformation energy, both of which originate from the large ultimate stress and strain of graphene, were the main reasons for the superior $E_{p}{ }^{*}$ in graphene.
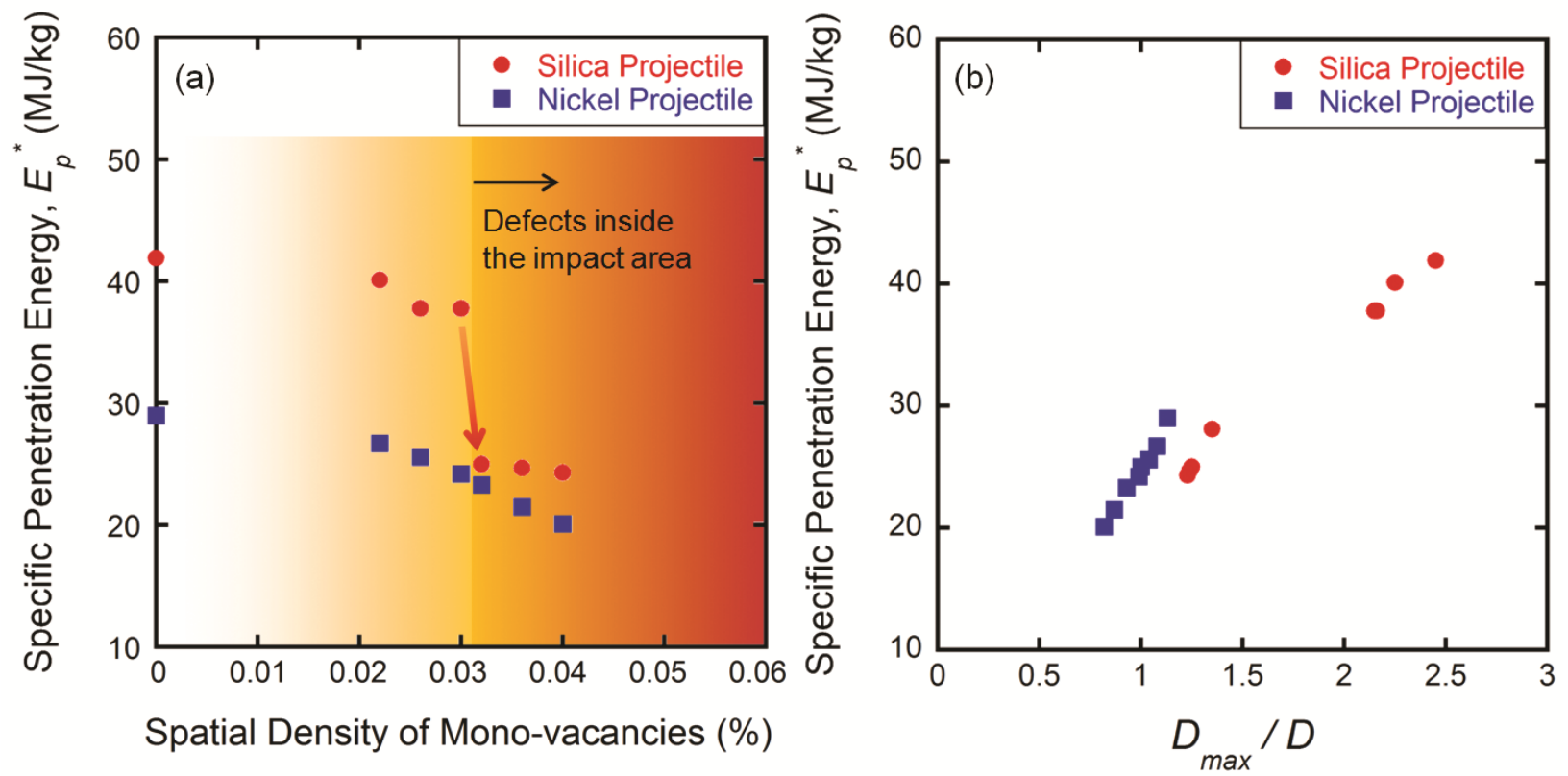

Figure 4. Effects of defects and the type of material used for the projectile on $E_{p}{ }^{*}$ : (a) Change in $E_{p}{ }^{*}$ with respect to the spatial density of mono-vacancies in graphene. Discontinuity in color, at 
$0.031 \%$ of defect density, indicates the introduction of mono-vacancies within the impact areas of both projectiles from that density of defects. In the penetration by the silica projectile, a significant decrease in $E_{p}{ }^{*}(\sim 42 \%)$ was observed when vacancies were introduced within the impact area. On the contrary, in the penetration by the nickel projectile, a monotonous decrease in $E_{p}{ }^{*}$ was observed as more vacancies were introduced, irrespective of their locations; (b) Change in $E_{p}{ }^{*}$ with respect to the maximum deformation diameter of graphene before initiation of the cracks $\left(D_{\max }\right)$ normalized by the diameter of the projectile $(D)$.

Defects inside the impact region in the penetration by the silica projectile resulted in much earlier initiation of cracks, leading to a reduction in the energy absorbed by the deformation of graphene, which, in turn, resulted in a significant decrease in $E_{p}{ }^{*}$. The lower $E_{p}{ }^{*}$ values in the penetration by the nickel projectile also can be explained by the less allowable deformation of graphene before the initiation of cracks, due to the weakened mechanical properties, which was caused by the graphene-nickel interactions. Tensile curves of graphene, shown in Fig. S6, prove the weakened mechanical properties of graphene when it is in contact with the nickel nanoparticle.

Figure 5 depicts some representative $E_{p}{ }^{*}$ values associated with graphene sheets with different types of defects, together with reported experimental trend for multilayer graphene [14]. We observed a significant decrease in $E_{p}{ }^{*}$ when the graphene sheets were weakened by defects. In the penetrations by the silica projectile, polycrystalline graphene with the grain size of $25 \AA$ had a $33 \%$ decrease in $E_{p}{ }^{*}$, compared with that of the pristine graphene. Description of the grain boundaries can be found in the supporting information. Also, the graphene with distributed vacancies within the impact area had a $42 \%$ decrease in $E_{p}{ }^{*}$ compared with that of the pristine graphene. In the penetrations by the nickel projectile, the decrease in $E_{p}{ }^{*}$ due to the defects was 
less significant. Polycrystalline graphene and the graphene with vacancies within the impact region had 8 and $\sim 20 \%$ decreases in $E_{p}{ }^{*}$, respectively. To date, graphene penetration experiments have been conducted with multilayer graphene using impact velocities of less than 1 $\mathrm{km} / \mathrm{s}$. Thus, our single-layer graphene penetration simulations using an impact velocity of $5 \mathrm{~km} / \mathrm{s}$ cannot be compared directly to the experimental values. In Fig. 5, the larger values of $E_{p}{ }^{*}$ obtained in this study can be explained as follows. First, the mechanical properties, such as ultimate stress and strain, of the single-layer graphene are superior to those of multilayer graphene; therefore resulting in the larger values of $E_{p}{ }^{*}$. Second, the penetration simulations in the nanoscopic regime entailed a significant deformation of the projectiles, which was contrary to the experiments. Since $A_{s}$ significantly changes as the projectile deforms, normalization of $E_{p}$ by the mass of graphene within $A_{s}$ could have been the other source of the difference. Furthermore, the potential used in this study might have overestimated $E_{p}{ }^{*}$ values.

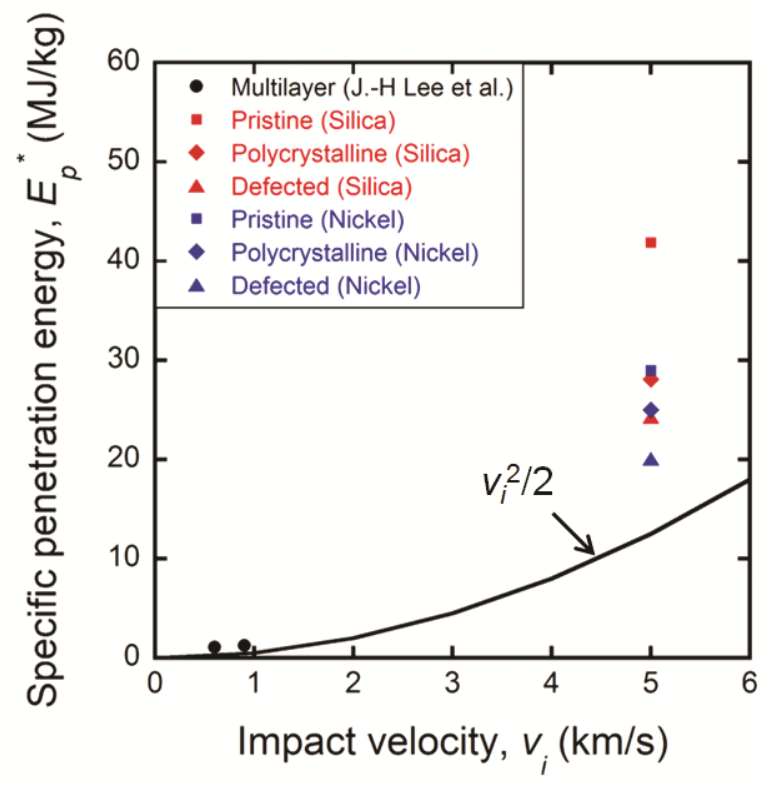

Figure 5. Representative $E_{p}{ }^{*}$ values obtained in this study, marked by red for penetrations by the silica projectile and blue for penetrations by the nickel projectile; they were indicated together with the experimental trend, marked by black dots for experimental values and black line for 
$v_{i}{ }^{2} / 2$, reported by Lee et al. [14]. $E_{P}{ }^{*}$ is expressed as $E_{P}{ }^{*}=v_{i}{ }^{2} / 2+E_{d}{ }^{*}$, where $v_{i}$ is the initial velocity of a projectile and $E_{d}{ }^{*}$ is the figure of merit to evaluate the delocalization ability of the impact energy [14].

\section{Conclusion}

We studied the mechanical behavior of graphene under the impact of nanoprojectiles on graphene by using ReaxFF reactive potential. We analyzed the atomistic features regarding the fracture of graphene induced by the discharge of projectiles made of different materials. The preferential edges of the cracks seem to be affected by the deformability of graphene before the formation of the cracks was initiated. It appears that less deformation before the initiation of the cracks led to more zigzag edges, whereas a large allowed deformation led to more armchair edges. In the penetrations by both the silica and the nickel projectiles, pairs of 5/7 defects were found at the edges of the cracks, and seven-member rings almost always were located closer to the tips of the cracks compared to five-member rings. We also addressed the effects on $E_{p}{ }^{*}$ that resulted from defects and the types of materials used to make the projectile. While a significant decrease in $E_{p}{ }^{*}(\sim 42 \%)$ was observed in the penetration by the silica projectile when defects were present within the impact area, a monotonic decrease in $E_{p}{ }^{*}$ was observed in the penetration by the nickel projectile as the number of mono-vacancies increased, irrespective of the location of vacancies. It seems that, based on Fig. 4(b), a large maximum allowable deformation before the initiation of the cracks and a large amount of energy absorbed during the deformation, both of which resulted from the superior ultimate stress and strain of graphene, are critical factors that contribute to the high $E_{p}{ }^{*}$ of graphene. The lower values of $E_{p}{ }^{*}$ in the penetration by the nickel projectile were explained by the weakening of the graphene due to its interaction with nickel. 
Further study on the analysis of crack development in the polycrystalline graphene will be able to provide more valuable information on the nanoparticle-impact study. Additionally, the study on the impact velocity that is required for the penetration of the polycrystalline graphene with different grain sizes seems to be an interesting research topic. Also, it may be beneficial to provide statistical information on the penetration-induced fractures and to study the effect of thickness on the mechanical behavior of graphene under the impact of nanoparticles. 
Acknowledgment. This research was supported by funding through AFOSR FA9550-11-10158 and by funding from the Fluid Interface Reactions, Structures and Transport (FIRST) Center, an Energy Frontier Research Center funded by the U.S. Department of Energy, Office of Science, Office of Basic Energy Sciences.

Supporting Information: Atomistic structures of graphene obtained from the penetration simulations, including the structures before the initiations of the cracks and after the penetrations. Tensile curves of pristine graphene and graphene in contact with the nickel nanoparticle. Descriptions of polycrystalline graphene and crack development in the polycrystalline graphene. 


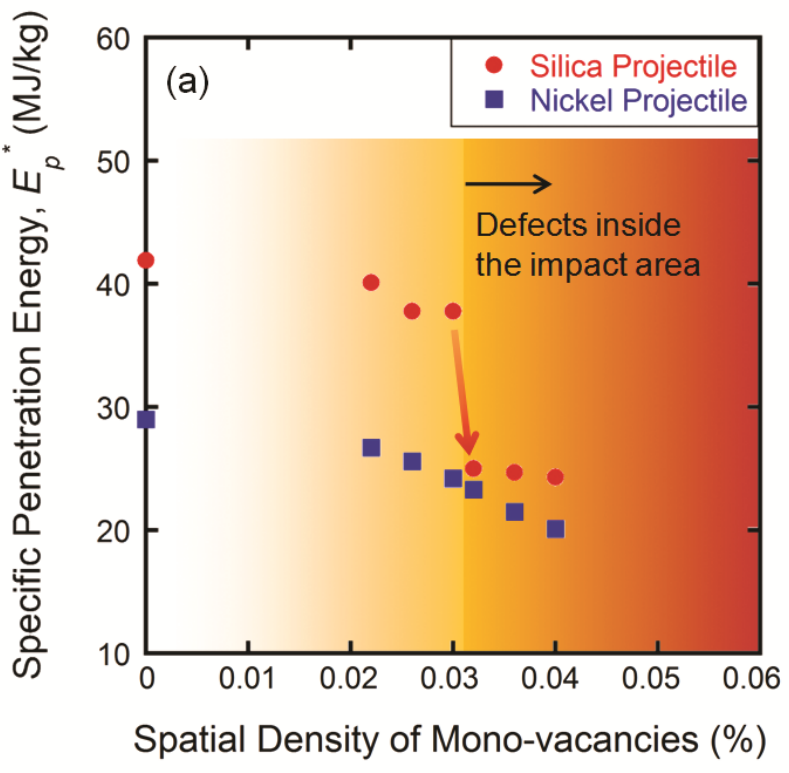

Cover figures 


\section{REFERENCES}

1. Novoselov, K. S.; Geim, A. K.; Morozov, S. V.; Jiang, D.; Zhang, Y.; Dubonos, S. V., et al. Electric Field Effect in Atomically Thin Carbon Films. Science 2004, 306, (5696), 666-669.

2. Geim, A. K.; Novoselov, K. S. The rise of graphene. Nat Mater 2007, 6, (3), 183-191.

3. Novoselov, K. S.; Jiang, Z.; Zhang, Y.; Morozov, S. V.; Stormer, H. L.; Zeitler, U., et al. Room-Temperature Quantum Hall Effect in Graphene. Science 2007, 315, (5817), 1379.

4. Geim, A. K. Graphene: Status and Prospects. Science 2009, 324, (5934), 1530-1534.

5. Lee, C.; Wei, X.; Kysar, J. W.; Hone, J. Measurement of the Elastic Properties and Intrinsic Strength of Monolayer Graphene. Science 2008, 321, (5887), 385-388.

6. Bolotin, K. I.; Sikes, K. J.; Jiang, Z.; Klima, M.; Fudenberg, G.; Hone, J., et al. Ultrahigh electron mobility in suspended graphene. Solid State Communications 2008, 146, (9-10), 351355.

7. Balandin, A. A.; Ghosh, S.; Bao, W.; Calizo, I.; Teweldebrhan, D.; Miao, F., et al. Superior Thermal Conductivity of Single-Layer Graphene. Nano Lett. 2008, 8, (3), 902-907.

8. Balandin, A. A. Thermal properties of graphene and nanostructured carbon materials. Nat Mater 2011, 10, (8), 569-581.

9. Yoon, K.; Hwang, G.; Chung, J.; Kim, H. g.; Kwon, O.; Kihm, K. D., et al. Measuring the thermal conductivity of residue-free suspended graphene bridge using null point scanning thermal microscopy. Carbon 2014, 76, (0), 77-83.

10. Meyer, J. C.; Geim, A. K.; Katsnelson, M. I.; Novoselov, K. S.; Booth, T. J.; Roth, S. The structure of suspended graphene sheets. Nature 2007, 446, (7131), 60-63.

11. Gong, Y.; Fei, H.; Zou, X.; Zhou, W.; Yang, S.; Ye, G., et al. Boron- and NitrogenSubstituted Graphene Nanoribbons as Efficient Catalysts for Oxygen Reduction Reaction. Chemistry of Materials 2015, 27, (4), 1181-1186.

12. Achtyl, J. L.; Unocic, R. R.; Xu, L.; Cai, Y.; Raju, M.; Zhang, W., et al. Aqueous proton transfer across single-layer graphene. Nat Commun 2015, 6.

13. Rafiee, M. A.; Rafiee, J.; Srivastava, I.; Wang, Z.; Song, H.; Yu, Z.-Z., et al. Fracture and Fatigue in Graphene Nanocomposites. Small 2010, 6, (2), 179-183.

14. Lee, J. H.; Loya, P. E.; Lou, J.; Thomas, E. L. Dynamic mechanical behavior of multilayer graphene via supersonic projectile penetration. Science 2014, 346, (6213), 1092-1096.

15. Lee, J.-H.; Veysset, D.; Singer, J. P.; Retsch, M.; Saini, G.; Pezeril, T., et al. High strain rate deformation of layered nanocomposites. Nat Commun 2012, 3, 1164.

16. Ozden, S.; Autreto, P. A. S.; Tiwary, C. S.; Khatiwada, S.; Machado, L.; Galvao, D. S., et al. Unzipping Carbon Nanotubes at High Impact. Nano Lett. 2014, 14, (7), 4131-4137.

17. Eller, M. J.; Liang, C.-K.; Della-Negra, S.; Clubb, A. B.; Kim, H.; Young, A. E., et al. Hypervelocity nanoparticle impacts on free-standing graphene: A sui generis mode of sputtering. The Journal of Chemical Physics 2015, 142, (4), 044308.

18. Zhang, Z.; Kutana, A.; Yakobson, B. I. Edge reconstruction-mediated graphene fracture. Nanoscale 2015, 7, (6), 2716-2722.

19. Topsakal, M.; Ciraci, S. Elastic and plastic deformation of graphene, silicene, and boron nitride honeycomb nanoribbons under uniaxial tension: A first-principles density-functional theory study. Physical Review B 2010, 81, (2), 024107.

20. Zhao, H.; Min, K.; Aluru, N. R. Size and Chirality Dependent Elastic Properties of Graphene Nanoribbons under Uniaxial Tension. Nano Lett. 2009, 9, (8), 3012-3015. 
21. Marianetti, C. A.; Yevick, H. G. Failure Mechanisms of Graphene under Tension. Physical Review Letters 2010, 105, (24), 245502.

22. Zhang, P.; Ma, L.; Fan, F.; Zeng, Z.; Peng, C.; Loya, P. E., et al. Fracture toughness of graphene. Nat Commun 2014, 5.

23. Zhang, Z.; Yang, Y.; Xu, F.; Wang, L.; Yakobson, B. I. Unraveling the Sinuous Grain Boundaries in Graphene. Advanced Functional Materials 2015, 25, (3), 367-373.

24. Song, Z.; Artyukhov, V. I.; Wu, J.; Yakobson, B. I.; Xu, Z. Defect-Detriment to Graphene Strength Is Concealed by Local Probe: The Topological and Geometrical Effects. ACS Nano 2015, 9, (1), 401-408.

25. Li, C.; Koslowski, M.; Strachan, A. Engineering Curvature in Graphene Ribbons Using Ultrathin Polymer Films. Nano Lett. 2014, 14, (12), 7085-7089.

26. Jung, G.; Qin, Z.; Buehler, M. J. Molecular mechanics of polycrystalline graphene with enhanced fracture toughness. Extreme Mechanics Letters 2015, 2, 52-59.

27. Qin, Z.; Taylor, M.; Hwang, M.; Bertoldi, K.; Buehler, M. J. Effect of Wrinkles on the Surface Area of Graphene: Toward the Design of Nanoelectronics. Nano Lett. 2014, 14, (11), 6520-6525.

28. Zang, J.; Ryu, S.; Pugno, N.; Wang, Q.; Tu, Q.; Buehler, M. J., et al. Multifunctionality and control of the crumpling and unfolding of large-area graphene. Nat Mater 2013, 12, (4), 321325.

29. Kim, K.; Artyukhov, V. I.; Regan, W.; Liu, Y. Y.; Crommie, M. F.; Yakobson, B. I., et al. Ripping Graphene: Preferred Directions. Nano Lett. 2012, 12, (1), 293-297.

30. Huang, X.; Yang, H.; van Duin, A. C. T.; Hsia, K. J.; Zhang, S. L. Chemomechanics control of tearing paths in graphene. Physical Review B 2012, 85, (19).

31. Sen, D.; Novoselov, K. S.; Reis, P. M.; Buehler, M. J. Tearing Graphene Sheets From Adhesive Substrates Produces Tapered Nanoribbons. Small 2010, 6, (10), 1108-1116.

32. Santos, R. P. B. d.; Perim, E.; Autreto, P. A. S.; Gustavo, B.; Galvão, D. S. On the unzipping of multiwalled carbon nanotubes. Nanotechnology 2012, 23, (46), 465702.

33. Jack, R.; Sen, D.; Buehler, M. J. Graphene Nanocutting Through Nanopatterned Vacancy Defects. Journal of Computational and Theoretical Nanoscience 2010, 7, (2), 354-359.

34. Zhang, S. L.; Mielke, S. L.; Khare, R.; Troya, D.; Ruoff, R. S.; Schatz, G. C., et al. Mechanics of defects in carbon nanotubes: Atomistic and multiscale simulations. Physical Review $B$ 2005, 71, (11).

35. Jensen, B. D.; Wise, K. E.; Odegard, G. M. The effect of time step, thermostat, and strain rate on ReaxFF simulations of mechanical failure in diamond, graphene, and carbon nanotube. Journal of Computational Chemistry 2015, 36, (21), 1587-1596.

36. Donald, W. B.; Olga, A. S.; Judith, A. H.; Steven, J. S.; Boris, N.; Susan, B. S. A secondgeneration reactive empirical bond order (REBO) potential energy expression for hydrocarbons. Journal of Physics: Condensed Matter 2002, 14, (4), 783.

37. Stuart, S. J.; Tutein, A. B.; Harrison, J. A. A reactive potential for hydrocarbons with intermolecular interactions. The Journal of Chemical Physics 2000, 112, (14), 6472-6486.

38. Srinivasan, S. G.; van Duin, A. C. T.; Ganesh, P. Development of a ReaxFF Potential for Carbon Condensed Phases and Its Application to the Thermal Fragmentation of a Large Fullerene. Journal of Physical Chemistry A 2015, 119, (4), 571-580.

39. Kroes, J. M. H.; Pietrucci, F.; van Duin, A. C. T.; Andreoni, W. Atom Vacancies on a Carbon Nanotube: To What Extent Can We Simulate their Effects? Journal of Chemical Theory and Computation 2015, 11, (7), 3393-3400. 
40. van Duin, A. C. T.; Dasgupta, S.; Lorant, F.; Goddard, W. A. ReaxFF: A Reactive Force Field for Hydrocarbons. The Journal of Physical Chemistry A 2001, 105, (41), 9396-9409.

41. Chenoweth, K.; van Duin, A. C. T.; Goddard, W. A. ReaxFF Reactive Force Field for Molecular Dynamics Simulations of Hydrocarbon Oxidation. The Journal of Physical Chemistry A 2008, 112, (5), 1040-1053.

42. Aktulga, H. M.; Fogarty, J. C.; Pandit, S. A.; Grama, A. Y. Parallel reactive molecular dynamics: Numerical methods and algorithmic techniques. Parallel Comput. 2012, 38, (4-5), 245-259.

43. Newsome, D. A.; Sengupta, D.; Foroutan, H.; Russo, M. F.; van Duin, A. C. T. Oxidation of Silicon Carbide by O2 and H2O: A ReaxFF Reactive Molecular Dynamics Study, Part I. The Journal of Physical Chemistry C 2012, 116, (30), 16111-16121.

44. Mueller, J. E.; van Duin, A. C. T.; Goddard, W. A. Development and Validation of ReaxFF Reactive Force Field for Hydrocarbon Chemistry Catalyzed by Nickel. The Journal of Physical Chemistry C 2010, 114, (11), 4939-4949.

45. Fogarty, J. C.; Aktulga, H. M.; Grama, A. Y.; van Duin, A. C. T.; Pandit, S. A. A reactive molecular dynamics simulation of the silica-water interface. The Journal of Chemical Physics 2010, 132, (17), 174704.

46. Zou, C.; Shin, Y. K.; van Duin, A. C. T.; Fang, H.; Liu, Z.-K. Molecular dynamics simulations of the effects of vacancies on nickel self-diffusion, oxygen diffusion and oxidation initiation in nickel, using the ReaxFF reactive force field. Acta Materialia 2015, 83, (0), 102112.

47. Lee, G.-D.; Wang, C. Z.; Yoon, E.; Hwang, N.-M.; Kim, D.-Y.; Ho, K. M. Diffusion, Coalescence, and Reconstruction of Vacancy Defects in Graphene Layers. Physical Review Letters 2005, 95, (20), 205501.

48. Trevethan, T.; Latham, C. D.; Heggie, M. I.; Briddon, P. R.; Rayson, M. J. Vacancy diffusion and coalescence in graphene directed by defect strain fields. Nanoscale 2014, 6, (5), 2978-2986.

49. Terdalkar, S. S.; Huang, S.; Yuan, H. Y.; Rencis, J. J.; Zhu, T.; Zhang, S. L. Nanoscale fracture in graphene. Chemical Physics Letters 2010, 494, (4-6), 218-222.

50. Koskinen, P.; Malola, S.; Häkkinen, H. Self-Passivating Edge Reconstructions of Graphene. Physical Review Letters 2008, 101, (11), 115502. 